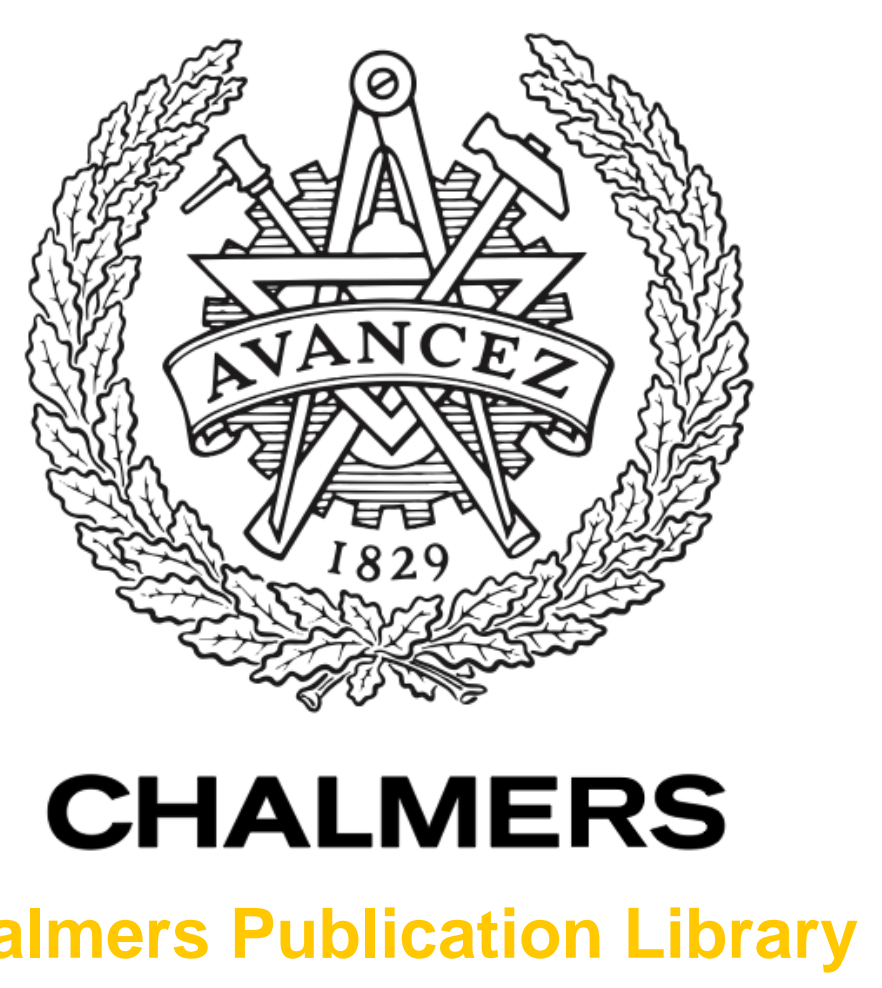

Chalmers Publication Library

\author{
Efficiency, Correlation, and Diversity Gain of UWB Multiport Self-Grounded Bow- \\ Tie Antenna in Rich Isotropic Multipath Environment
}

This document has been downloaded from Chalmers Publication Library $(\mathrm{CPL})$. It is the author's version of a work that was accepted for publication in:

9th International Workshop on Antenna Technology (iWAT). Karlsruhe, GERMANY. MAR 0406, 2013

Citation for the published paper:

Hussain, A. ; Kildal, P. ; Al-Rawi, A. (2013) "Efficiency, Correlation, and Diversity Gain of UWB Multiport Self-Grounded Bow-Tie Antenna in Rich Isotropic Multipath Environment". 9th International Workshop on Antenna Technology (iWAT). Karlsruhe, GERMANY. MAR 04-06, 2013 pp. 336-339.

Downloaded from: http://publications.lib.chalmers.se/publication/182076

Notice: Changes introduced as a result of publishing processes such as copy-editing and formatting may not be reflected in this document. For a definitive version of this work, please refer to the published source. Please note that access to the published version might require a subscription. 


\title{
Efficiency, Correlation, and Diversity Gain of UWB Multiport Self-Grounded Bow-Tie Antenna in Rich Isotropic Multipath Environment
}

\author{
A. Hussain ${ }^{*(1)}$, P-S. Kildal ${ }^{(1)}$, A. Al-Rawi ${ }^{(1)}$, and J. Yang ${ }^{(1,2)}$ \\ (1) Antenna Group, Department of Signals \& Systems, Chalmers University of Technology, Gothenburg, Sweden \\ Email: ahmed.hussain@,chalmers.se
}

(2) GAP Waves AB, Gothenburg, Sweden

\begin{abstract}
The paper studies the performance of a novel ultra-wideband multiport self-grounded bow-tie antenna and presents simulated embedded radiation efficiencies and correlation at each antenna port, and the diversity gain of the antenna in rich isotropic multipath environment.
\end{abstract}

\section{INTRODUCTION}

Multiport antennas are needed in wireless communications systems using Multiple-input Multiple-output (MIMO) technology to increase the throughput or channel capacity. The embedded radiation efficiencies and the correlation between the antenna ports are main factors determining the diversity gain. Recently it has been shown that the diversity gain (previously defined at 1\% CDF-level in [1][2]), represents an equally large improvement at $99 \%$ throughput-level [3], in a rich isotropic multipath (RIMP) environment.

The four port self-grounded bow-tie antenna [4]-[8] is a compact ultra-wideband (UWB) MIMO antenna that has been recently developed at the Antenna Group, Chalmers University of Technology; see Fig. 1. This newly developed antenna provides unique features. Besides its compact geometry, the antenna has ultra-wideband frequency of operation, i.e. $0.5-16 \mathrm{GHz}$. The simulations show that the reflection coefficient is below $-7 \mathrm{~dB}$, the mutual coupling below $-12.5 \mathrm{~dB}$, and the total embedded radiation efficiency above $-1 \mathrm{~dB}$, over the whole bandwidth; see Fig. 2-3.

This antenna is mainly developed for Bluetest reverberation chambers but it can be also used in other applications, e.g. micro base-stations. It is based on the idea of self-grounded monopoles. It is designed using Copper material in CST Microwave Studio and its performance is optimized using genetic algorithm. The antenna has a simple mechanical construction that is easy to manufacture.

To characterize the performance of the four-port self-grounded bow-tie antenna, we extract S-parameters and embedded radiation patterns of each antenna port from CST Microwave Studio for the frequency range of 0.5-16 GHz. From the S-parameters we can calculate the embedded radiation efficiency and total embedded radiation efficiency at each port. The simulated embedded far field functions from CST are then exposed to RIMP environment to calculate correlation and diversity gain. This is done by using a ray-based simulation tool called ViRM-lab. The RIMP environment is emulated by reverberation chambers as explain in [3].

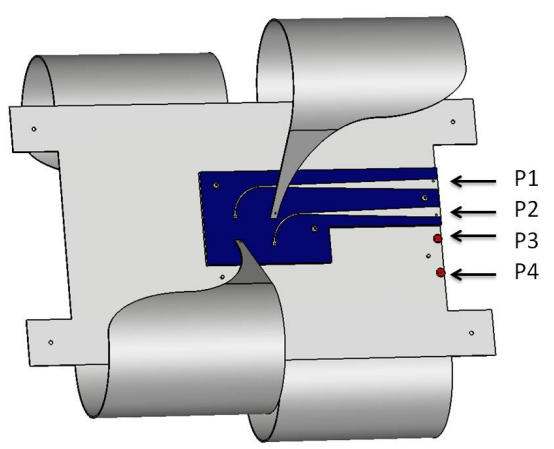

Fig. 1: CST model of 4-port self-grounded bow-tie antenna 


\section{RADIATION EFFICIENCY AND CORRELATION USING S-PARAMETERS}

We simulate the multiport antenna structure in CST Microwave Studio to get S-parameters between all ports for the whole frequency range; see Fig. 2. For the lossless antenna, the embedded radiation efficiency, the total embedded radiation efficiency and the correlation in RIMP environment can be formulated in terms of the S-parameters.

The embedded radiation efficiency is defined in [2]. The formulas in terms of S-parameters for embedded radiation efficiency $e_{\text {rad }}$ and total embedded radiation efficiency $e_{\text {rad }}$ oftal of embedded element number $i$ when there are total $N$ elements, are shown below in (1) and (2) respectively:

$$
\begin{gathered}
e_{\text {rad }}=\frac{1-\sum_{j=1}^{N}\left|S_{i j}\right|^{2}}{1-\left|S_{i i}\right|^{2}} ; e_{r e f l}=1-\left|S_{i i}\right|^{2} \\
e_{\text {rad } d_{\text {total }}}=e_{\text {rad }} * e_{\text {refl }}=1-\sum_{j=1}^{N}\left|S_{i j}\right|^{2}
\end{gathered}
$$

where $e_{\text {refl }}$ denotes mismatch efficiency. Since all antenna petals are symmetrical or anti-symmetrical with respect to each other, they have the same radiation efficiency as shown in Fig. 3. The results plotted in Fig. 3 show comparison of efficiencies which are calculated by using CST itself and by using simulated S-parameters from CST. The results from both are quite similar at high frequencies i.e. above $1 \mathrm{GHz}$ but at $0.5 \mathrm{GHz}$ there are differences between the two calculations. We believe that the embedded radiation efficiency calculations using S-parameters from CST are more accurate. These simulated results can be validated by the measurement results of this antenna.

The correlation between the ports in a RIMP environment is very low, smaller than 0.1 , due to the fact that mutual coupling between the ports is lower than $-10 \mathrm{~dB}$; see Fig. 4. The correlation can be calculated by using far-field functions [2] or S-parameters. The formula for calculating correlation $\rho$ in terms of S-parameters is shown in (3).

$$
\left.\rho_{i j}=\frac{S_{i i} S_{i j}+S_{j i} S_{j j}}{\sqrt{\left[1-\left(\left|S_{i i}\right|^{2}+\left|S_{i j}\right|^{2}\right)\right.}\left[1-\left(\left|S_{j i}\right|^{2}+\left|S_{j j}\right|^{2}\right)\right.}\right]
$$

The correlation results in Fig. 4 show that only at $0.5 \mathrm{GHz}$, there is difference in results using two different methods but for higher frequency range i.e. 1-16 GHz, the results look similar. Since we have more samples of S-parameters to show more accurate results compared to limited number of far-field functions due to complexity, we believe that the correlations calculated using S-parameters are more accurate than calculated using far-field functions.

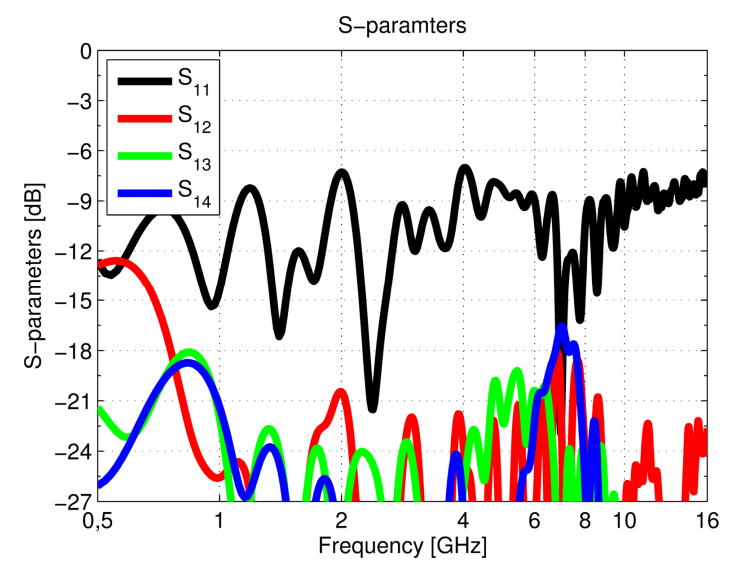

Fig. 2: Simulated S-parameters of 4-port self-grounded bow-tie antenna from CST. 

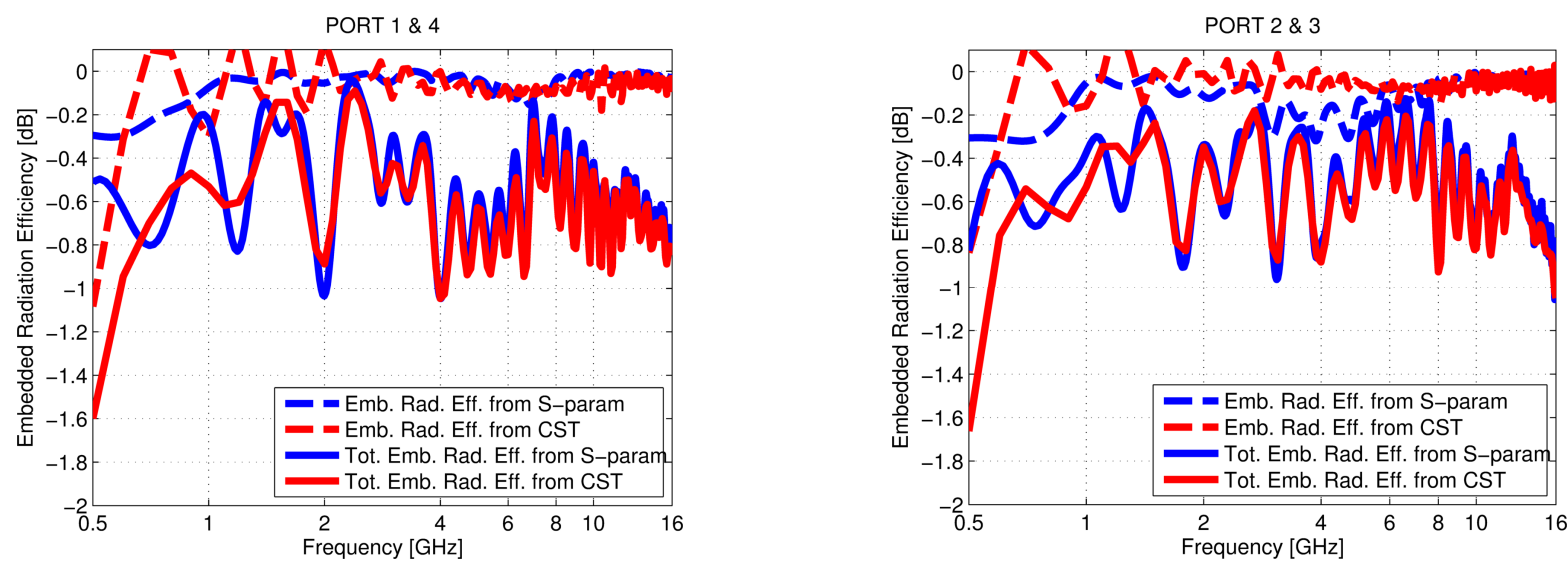

Fig. 3: Embedded radiation efficiencies of ports $1 \& 4$ (left) and ports $2 \& 3$ (right) calculated by CST (red color) and calculated by S-parameters from CST (blue color).
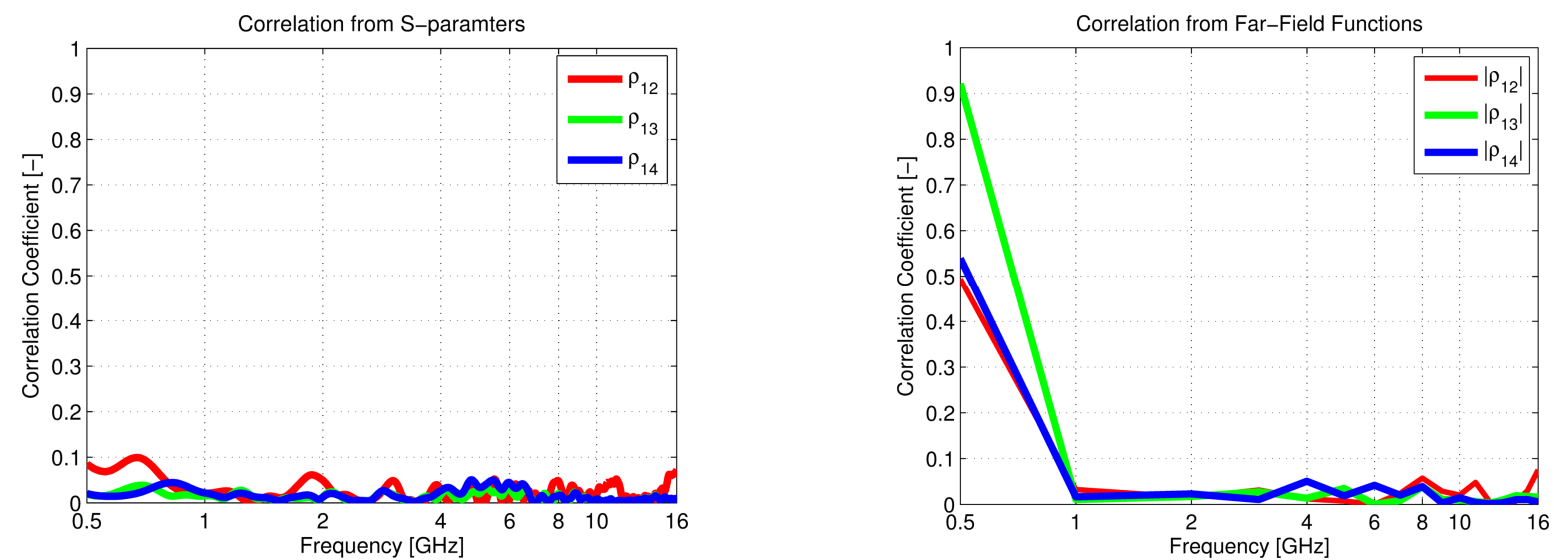

Fig. 4: Correlations using S-parameters (left) and using embedded far-field functions (right) between port 1 and port 2,3 , and 4 .

\section{DIVERSITY GAIN SIMULATIONS IN MULTIPATH ENVIRONMENT USING VIRM-LAB}
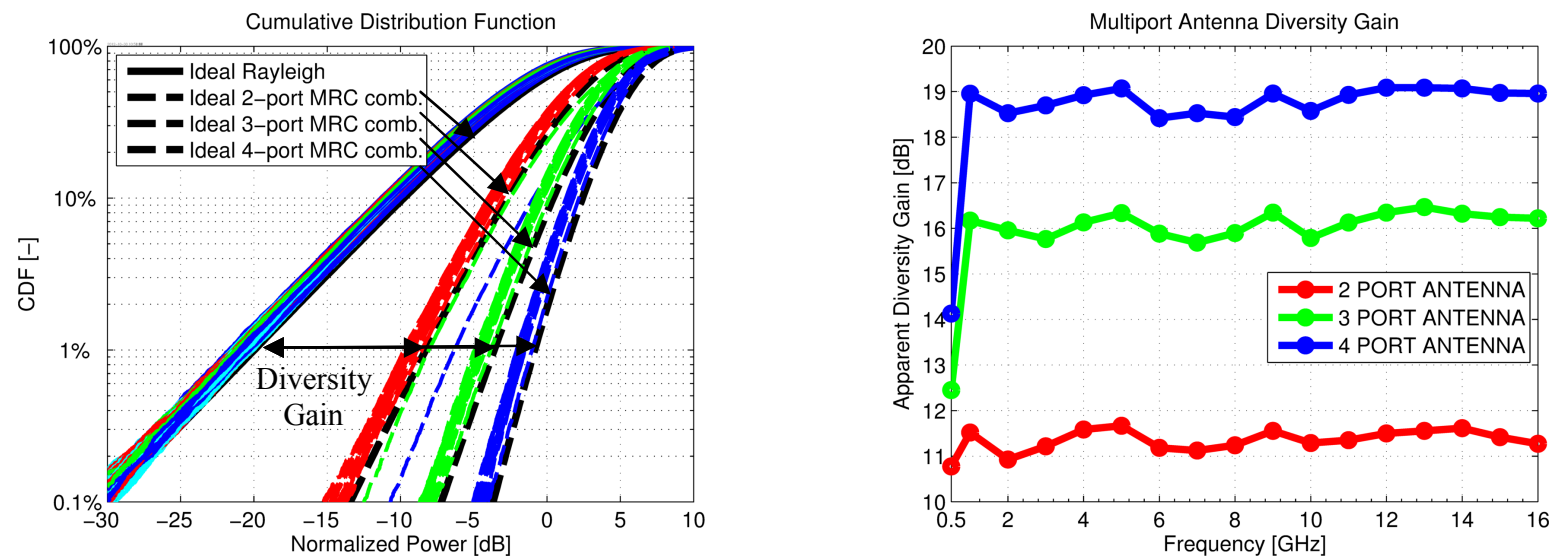

Fig. 5: CDF of each antenna port and 2, 3, and 4-port MRC combined diversity channel (left), and Apparent diversity gains at 1\% CDF-level over UWB for 2-port, 3-port, and 4-port diversity antenna (right).

The self-grounded bow-tie antenna can be used as a 2-, 3-, or 4-port MIMO antenna. Maximal ratio combining (MRC) scheme has been used to determine the diversity performance of this antenna. The antenna diversity gain in RIMP environment is obtained by simulations using a ray-based simulation tool called ViRM-lab [9]. We use a set of 20 
incoming waves having randomly distributed amplitude, phase, polarization, and angle of arrival (AoA). The amplitude is Rayleigh distributed while phase, polarization angle, and AoA are uniformly distributed in a 3D space. The antenna far-field patterns are exposed to these incoming waves which combine constructively and destructively, and result in a voltage sample at each antenna port. We collect 100,000 voltage samples at each antenna port by simulating 100,000 realizations of such a set of 20 incoming waves which are completely random.

Using these 100,000 complex-Gaussian distributed voltage samples from each antenna port, we can plot cumulative distribution function (CDF); see Fig. 5 (left). In RIMP environment, the diversity gain of an antenna is typically defined at $1 \%$ CDF-level. To evaluate diversity gain, we choose the optimal combination scheme i.e. MRC. The apparent diversity gain is defined as the difference of power-levels in $\mathrm{dB}$ between the CDFs of the antenna port with best efficiency and the diversity combined channel, typically at 1\% CDF-level; see Fig. 5 (left).

\section{CONCLUSIONS \& DISCUSSION}

The diversity gains calculated at 1\% CDF-level from Fig. 5 (left) are plotted in Fig. 5 (right) for the whole frequency range show that there is a clear improvement in the diversity gains of the antenna when we increase the spatial diversity from 2-ports to 3-ports and 4-ports diversity antenna. The improvement is seen over the whole wide bandwidth, which makes this antenna very useful for many practical applications. The antenna diversity gain is quite close to the ideal MRC-combined 2-port, 3-port, and 4-port diversity antennas i.e. $11.7 \mathrm{~dB}, 16.4 \mathrm{~dB}$, and $19.1 \mathrm{~dB}$ respectively. This is due to very high embedded efficiencies (Fig. 3) and very low cross-correlation between the antenna-ports (Fig. 4). At 0.5 $\mathrm{GHz}$, the apparent diversity gain is very low, see Fig. 5 (right). This is due to the fact that cross-correlation between the antenna ports is very high at $0.5 \mathrm{GHz}$, see Fig. 4 which is a direct result of high mutual coupling between the antenna ports at $0.5 \mathrm{GHz}$; see Fig. 2 .

\section{ACKNOWLEDGMENT}

This work has been supported in part by The Swedish Governmental Agency for Innovation Systems (VINNOVA) within the VINN Excellence Center Chase.

\section{REFERENCES}

[1] P.-S. Kildal and K. Rosengren, "Correlation and capacity of MIMO systems and mutual coupling, radiation efficiency and diversity gain of their antennas: Simulations and measurements in reverberation chamber", IEEE Communications Magazine, vol. 42, no. 12, pp. 102-112, Dec. 2004.

[2] K. Rosengren and P.-S. Kildal, "Radiation efficiency, correlation, diversity gain, and capacity of a six monopole antenna array for a MIMO system: Theory, simulation and measurement in reverberation chamber", Proceedings IEE, Microwave Antennas Propagation, vol. 152, no. 1, pp.7-16, February 2005. See also Erratum published in August 2006.

[3] P-S. Kildal, C. Orlenius, J. Carlsson, "OTA Testing in Multipath of Antennas and Wireless Devices With MIMO and OFDM," Proceedings of the IEEE, vol.100, no.7, pp.2145-2157, July 2012

[4] A. Al-Rawi, A New Compact Wideband MIMO Antenna for Reverberation Chambers, Master of Science Thesis, ISSN 99-2747920-4; Ex065/2012. Chalmers Publication Library, 2012.

[5] J. Yang, A. Kishk, "The self-grounded Bow-Tie antenna," Antennas and Propagation (APSURSI), 2011 IEEE International Symposium on, vol., no., pp.1452-1455, 3-8 July 2011

[6] J. Yang and A. Kishk, "A novel low-profile compact directional ultra-wideband antenna: the self-grounded BowTie antenna”, IEEE Trans. on Antennas Propag., vol. 60, no. 3, pp. 1214-1220, March 2012.

[7] Jian Yang, Ali Al-Rawi, Magnus Franzén, Charlie Orlenius, "Self-grounded Antenna Arrangement”, Swedish Patent Application SE-1251166-3, 2 October 2012.

[8] A. Al-Rawi, J. Yang, C. Orlenius and M. Franzen, "The double-sided 4-port Bow-tie antenna: a new compact wideband MIMO antenna”, 7th Eur. Conf. on Antennas Propagat. (EuCAP2013), Gothenburg, 8-12 April 2013.

[9] U. Carlberg, J. Carlsson, A. Hussain, P-S. Kildal, "Ray based multipath simulation tool for studying convergence and estimating ergodic capacity and diversity gain for antennas with given far-field functions," ICECom, 2010 Conference Proceedings, vol., no., pp.1-4, 20-23 Sept. 2010 